\title{
THE DIAGNOSIS AND TREATMENT OF MALIGNANT MELANOMA OF THE SKIN
}

\author{
D. C. Bodenham, F.R.C.S.E. \\ O. C. Lloyd, M.D. \\ From the Department of Plastic Surgery, Frenchay Hospital, and the Department of Pathology, \\ University of Bristol
}

ThAT malignant melanoma is not as black as it is painted is now becoming apparent. Never a common tumour, it has an incidence of 2 per 100,000 in the south-western region of England, where there is a population of three million, as compared with a rate of $4 \mathrm{r} .8$ for thoracic tumours. But the five-year survival rate in the same region is $46 \%$ for melanoma, which is far better than that of most of the common cancers (thoracic 5\%, stomach $10 \%$, breast $43 \%$ ).

The survival rates in different writers' series of cases of malignant melanoma are difficult to compare, ranging as they do from $20 \%$ to $58 \%$ (Petersen, Bodenham and Lloyd, 1962, Table V), but one surgeon (Pack, Scharnagel and Gerber, 1953; Pack, 1959) has shown an improvement over the years. One would like to be able to assume that any improvement was entirely due to the nature of the treatment, but the evidence seems to be that it is partly due to the composition of the sample.

It has, for instance, been shown by most writers but denied by some (e.g. Block and Hartwell, 196r) that the prognosis is twice as good in women as in men. But the number of men and women in different series varies greatly. In America the sexes are usually equal or (as in two recent studies, Ochsner and Harpole, 1962; and Block and Hartwell, I96r) there is a slight preponderance of men. But in England there is sometimes a $2:$ I female preponderance (Petersen and others, 1962), and this holds good for Denmark also (Clemmesen and Schulz, I960, 59.4\%; Olsen, I961, 66\%). Unless malignant melanoma is a different disease on the other side of the Atlantic, which is unlikely, this can only mean that half the women in America suffering from malignant melanoma are not going to the great clinics for treatment. If they did, the prognosis for this disease as a whole would be better in the American series than it is.

Another thing making for variable results is the fact that melanomas in some sites have a better prognosis than those in others. Petersen and others (1962) have shown that favourable sites are the legs of women under the age of 40 and the faces of elderly women. The latter are well known to have slowly developing growths, but their tendency to advance and spread has been proved. An example of an unfavourable site $\vec{s}$ the trunk, where $32.3 \%$ of melanomas have developed metastases when first seen by the surfu geon, as compared with $12.8 \%$ for the head and neck or $22.4 \%$ for the body as a whole. Bưf whereas melanoma of the head and neck accounted for $39.8 \%$ of their series of 220 cases, those of the trunk were only $14.2 \%$. In Ochsner and Harpole series (1962) the proportions are reversed: head and neck $22 \%$, trunk $35 \%$. Discrepancies suc\$ as these are bound to affect the overall five-yeas survival figures. To take the other example, melanoma of the leg is in the south-wester region of England, in Denmark and in White (1959) series from California much commoner if women than in men. As this is a site where melanoma has a favourable prognosis $(76 \%$ fieyear survival in the cases of Petersen and othas r 962), those series which do not contain a fat proportion of women are at a manifest disadvan tage.

The recent advances which have contributed to improved prognosis are:

(a) Early and precise diagnosis.

(b) Adequate primary treatment.

(c) Elimination of meddlesome interference.

\section{Diagnosis}

Malignant melanoma so often arises from $\stackrel{\frac{O}{3}}{\underline{3}}$ benign melanoma (lentigo or mole) that the dise tinction between the two is of the first importance The benign melanoma starts as a persistenf freckle or patch of pigmentation in which the melanocytes of the basal epidermis are hypero trophied and are producing an excess of melanin which they pass on to the epidermal cells. Nexo the melanocytes may proliferate to form buds in the epidermis (Fig. I). These are commonly seen in the moles of children and in the active moleg of adults. These epidermal buds of melanocytes migrate in an orderly fashion into the dermise where they become nævus cells. The deeper they? go the more fibrillary they become and they lose their power of melanogenesis. The neighbouring structures (hair follicles, blood vessels, nerves $\$$ hypertrophy and the result is the common hairy 


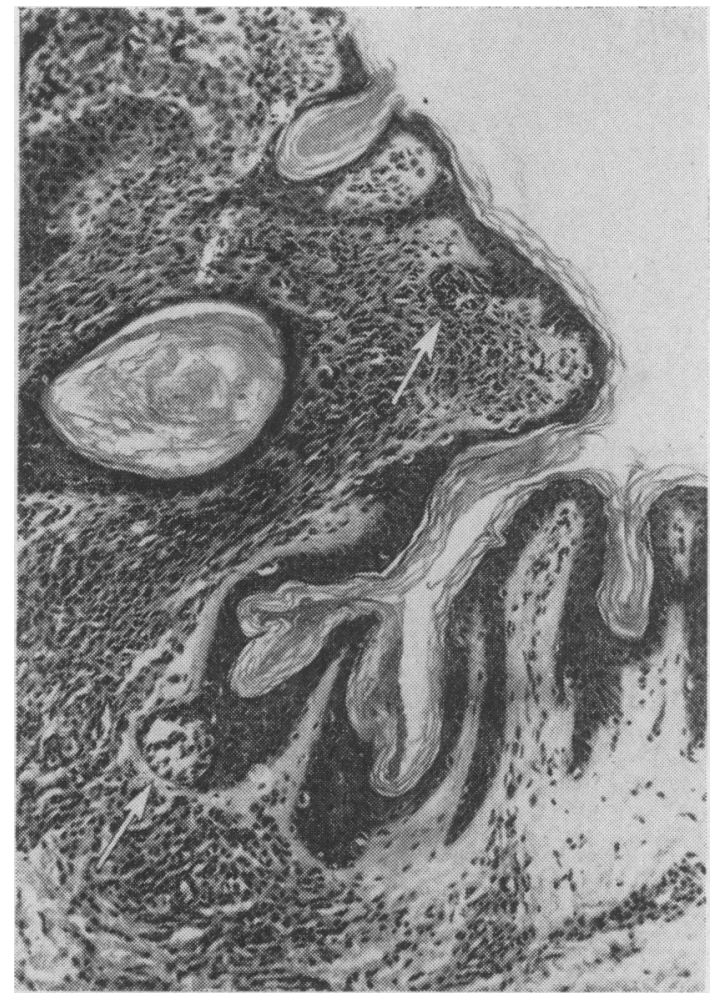

FIG. I.-Benign melanoma (hairy mole or compound nævus) in a child. The arrows point to an intraepidermal bud of melanocytes. (25/61 x 103).

mole or pigmented nævus (Fig. 2). In an adult mole the epidermal melanocytes usually stop budding, but may become reactivated again at any time. When malignancy takes place the change arises in these cells.

The signs of malignant change are these: the brown patch becomes larger. It may become darker, or-occasionally lighter, or develop a dark fringe. It irritates and may get rubbed. The overlying epidermis may become opaque and then weep, bleed or ulcerate with trivial injury. Above all, a tumour may develop in the pigmented flare. Microscopically, the malignant melanocytes will be seen (Fig. 3) to be much larger (up to eight times) and their invasion of the dermis is disorderly. Clumps of pigmented melanocytes may be seen migrating through the epidermis to be shed at the surface with the keratin (Fig. 4). With invasion there is inflammation and destruction of the dermis, and the larger the tumour masses become the more disorderly is the distribution of pigment.

\section{Errors of Diagnosis}

Errors of diagnosis are responsible for incorretc treatment, delay in referring cases for treatment,

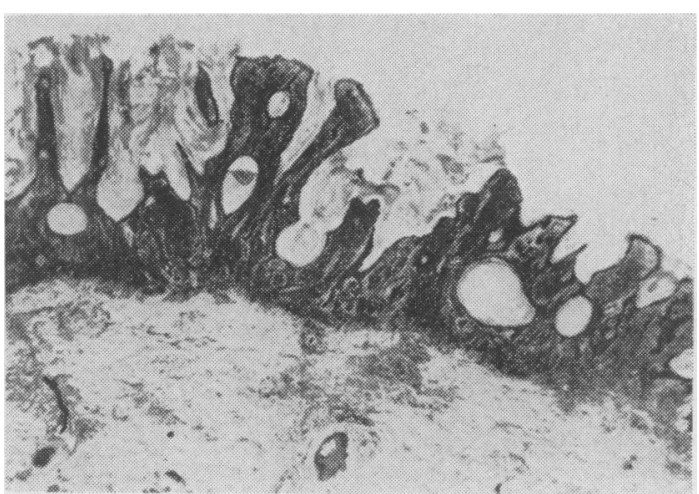

FIG. 2.-Benign melanoma (same tumour as Fig. I). Low magnification designed to show the orderliness of the invasion of dermis by melanocytes (nævus cells). They are mainly in the papillary layer, but where they invade the deep dermis they are around the hair follicles. $(25 / 6$ I x II $)$

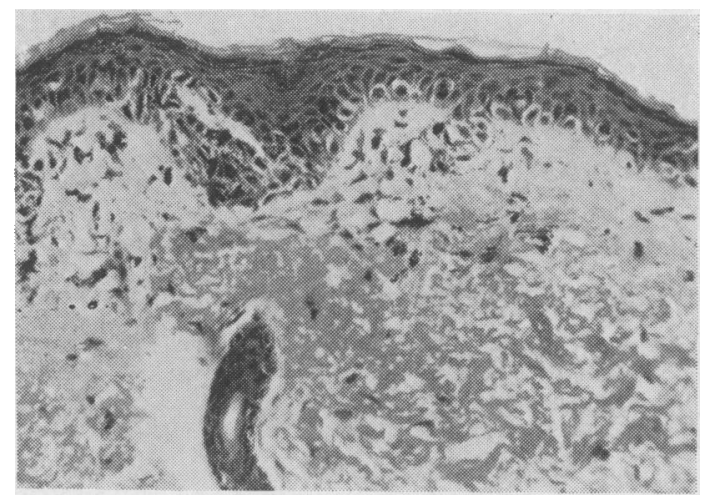

FIG. 3.-Malignant melanoma, Stage I. Large melanocytes form an irregular intra-epidermal bud. Compare with Fig. 8 and contrast with Fig. I. $(23 / 6$ I $\times 92)$

or may even prevent treatment altogether until it is too late. The errors most frequently encountered by us are listed below (Petersen and others, 1962).

\section{Errors by the Clinician}

(I) Malignant melanoma diagnosed as something else.

Presentation: Little or no pigment and nonulcerating. Diagnosed as epidermoid cyst, keloid, benign tumour of skin.

Presentation: Non-pigmented, rapidly growing, tense and tender. Diagnosed as acute infection, blind boil (Fig. 5).

Presentation: Ulcerating lesion. Diagnosed as pyogenic granuloma, whitlow (Fig. 6), epithelioma, ulcerating vascular nævus or hæmangioma.

(2) Other lesions diagnosed as malignant melanoma. 


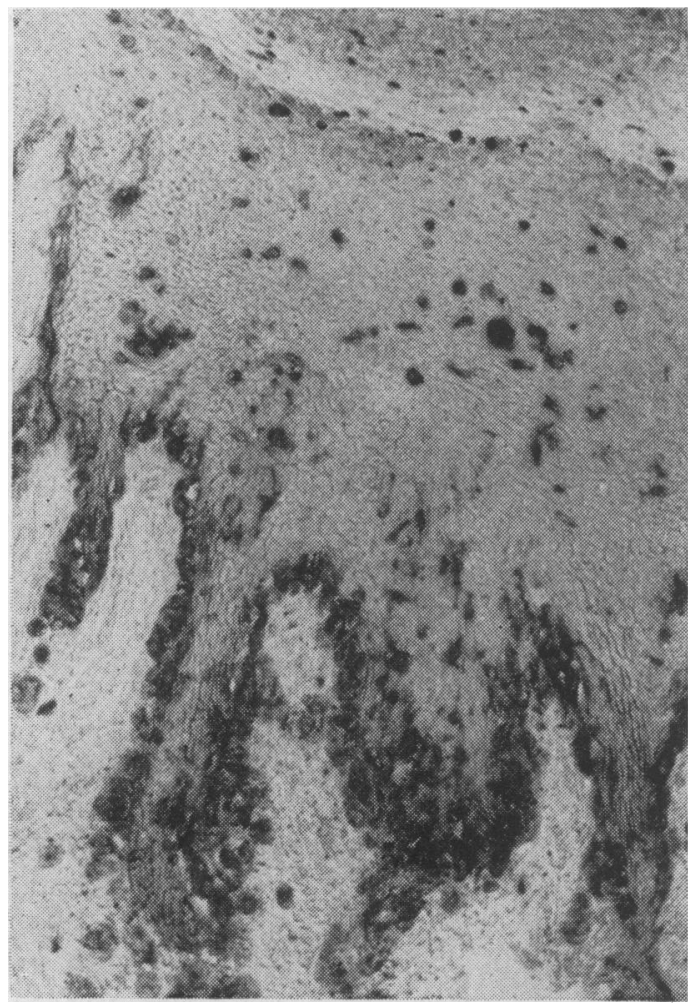

FIG. 4.-Frozen section, dopa impregnation. Edge of pigmented flare of Stage 3 malignant melanoma of foot. Malignant melanocytes not only invade dermis but are carried to the surface and shed with the keratin. (I64/52 $\times 55)$

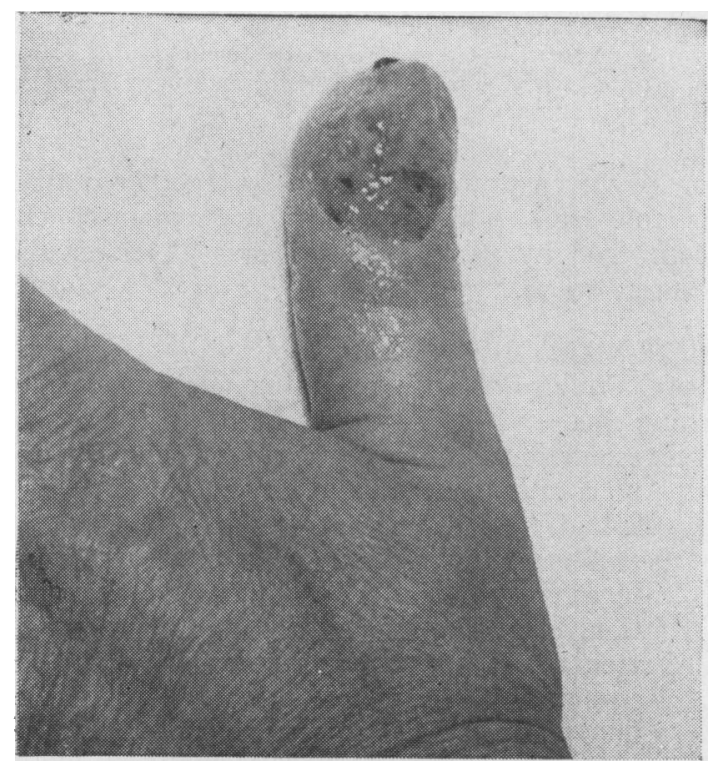

Fig. 6.-Non-pigmented ulcerating subungual malignant melanoma, mistaken elsewhere for a whitlow. (69/61, B. T.)

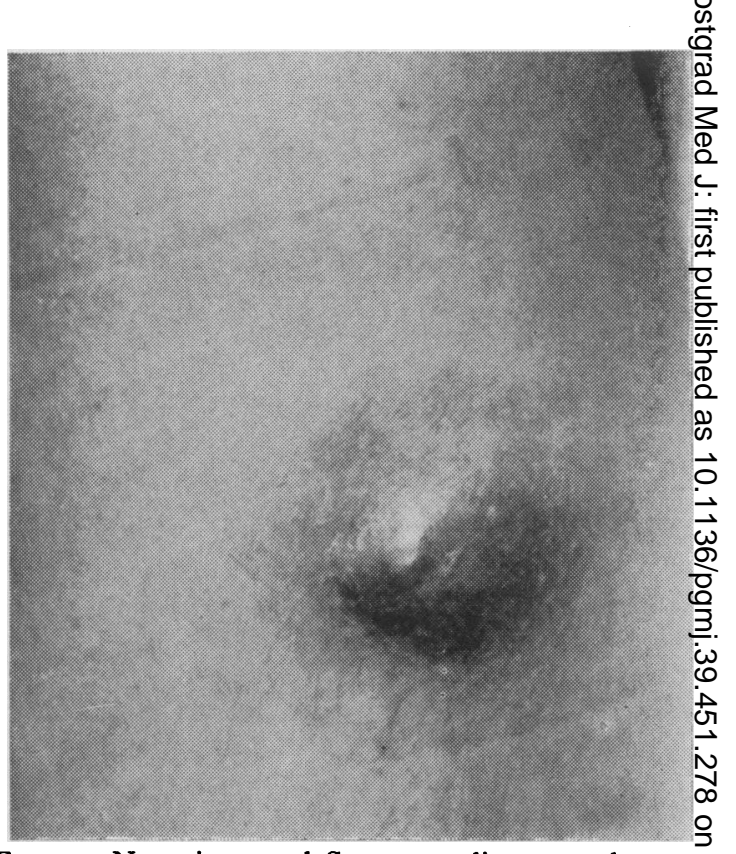

Fig. 5. - Non-pigmented Stage 3 malignant melanomaof leg, first mistaken for a 'blind boil', then for ans anaplastic carcinoma, on incision biopsy elsewhere The shady areas in the photograph are red, not brown. (126/60, E. V.)

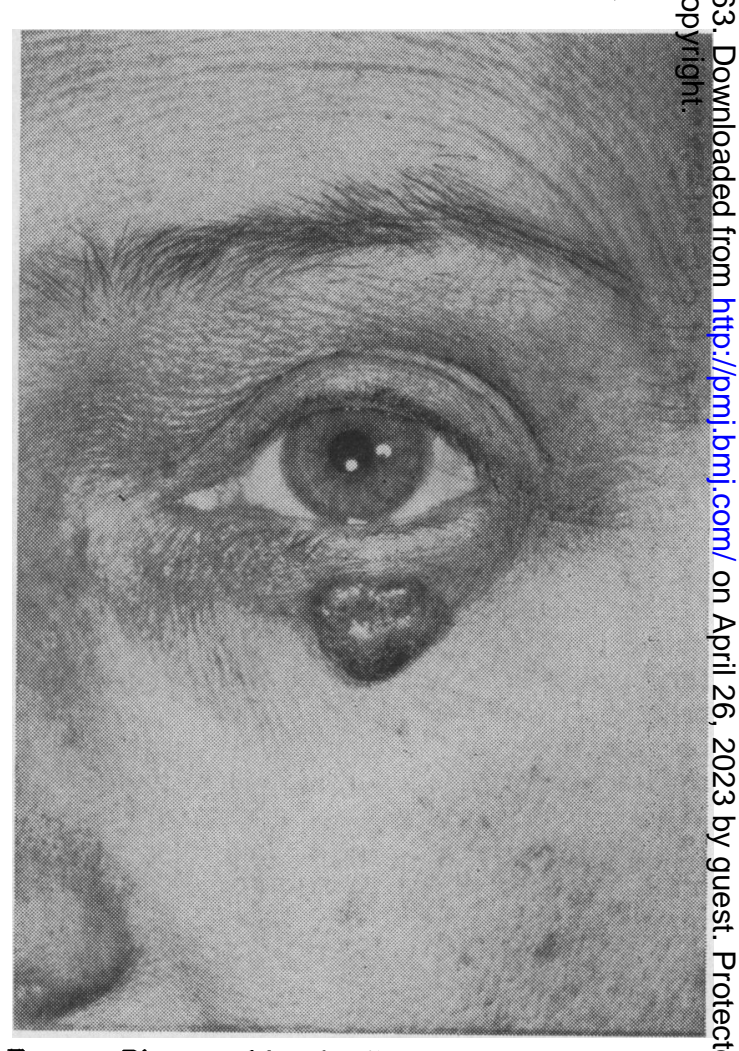

Fig. 7.-Pigmented basal-cell carcinoma. (1958, K. E.) $\overrightarrow{\mathbb{B}}$ 
Presentation: Pigmented lesion. True nature: Pigmented basal-cell carcinoma (Fig. 7), small thrombosed angioma, histiocytoma, pigmented papilloma or senile wart.

Presentation: Ulcerating lesion. True nature: Ulcerating hæmangioma.

\section{Errors by the Pathologist}

(I) Malignant melanoma diagnosed as something else. Diagnosed as:

(a) Benign melanoma (with all its synonyms, particularly 'junctional nævus').

(b) Squamous or anaplastic carcinoma.

(c) Malignant hæmangioma.

(2) Other lesions diagnosed as malignant melanoma. True nature:

(a) Benign melanoma, often with recent proliferation of epidermal melanocytes.

(b) Juvenile melanoma (Spitz, I948).

(c) Cellular variety of blue nævus (Allen and Spitz, 1953).

(d) Histiocytoma.

(e) Pigmented basal-cell carcinoma.

(f) Pigmented squamous papilloma, usually inflamed.

It will be helpful at this point to discuss some of these errors of diagnosis. The majority of clinical errors occur because the diagnosis of malignant melanoma was not thought of. The clinician therefore has a responsibility to enlist the help of a pathologist whenever doubt arises and it is fundamental that he should not fall into the trap of submitting a tiny sample of the tumour, often damaged by dissecting forceps so that the cell structure is distorted, a piece which is both too small to identify and may not represent the whole picture, so that the pathologist is at a great disadvantage and may well make a mistake in diagnosis, which could so easily be avoided. The total excision biopsy, with a small margin of clear tissue around the tumour, fulfils the necessary criteria by avoiding direct trauma to the tumour and by offering to the pathologist such material as he can reliably report on.

Epidermal Budding. One of the commonest errors of diagnosis is the misinterpretation of the buds of epidermal melanocytes found both in the benign and malignant melanomas. This has led to the use of the ambiguous term junctional nævus', which we do not use, but which is used by some pathologists as a convenient fence upon which to sit. The remedy is to study the behaviour of epidermal melanocytes in the pigmented flare which surrounds a tumorous malignant melanoma (Fig. 8). The tumour is invasive but the flare is pre-invasive, at least at its periphery. When the criteria so acquired are applied to a wholly non-invasive melanoma, the distinction between

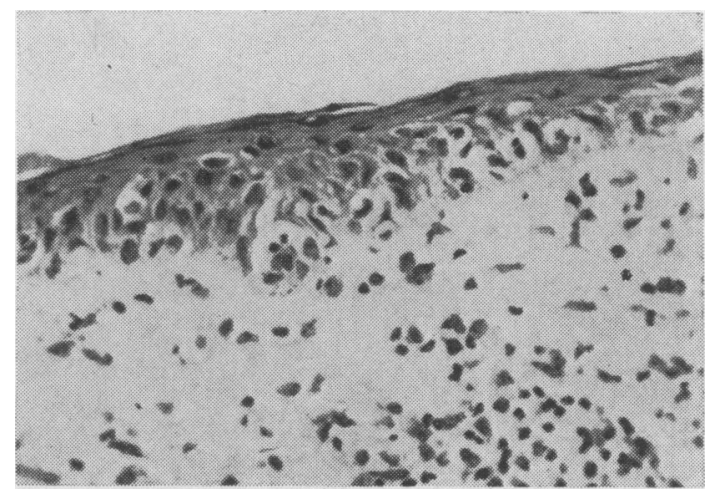

Fig. 8.-Malignant melanoma, Stage 3. Part of pigmented flare to show buds of melanocytes forming in epidermis and invading dermis, with reactive inflammation. $(91 / 51 \times 180)$

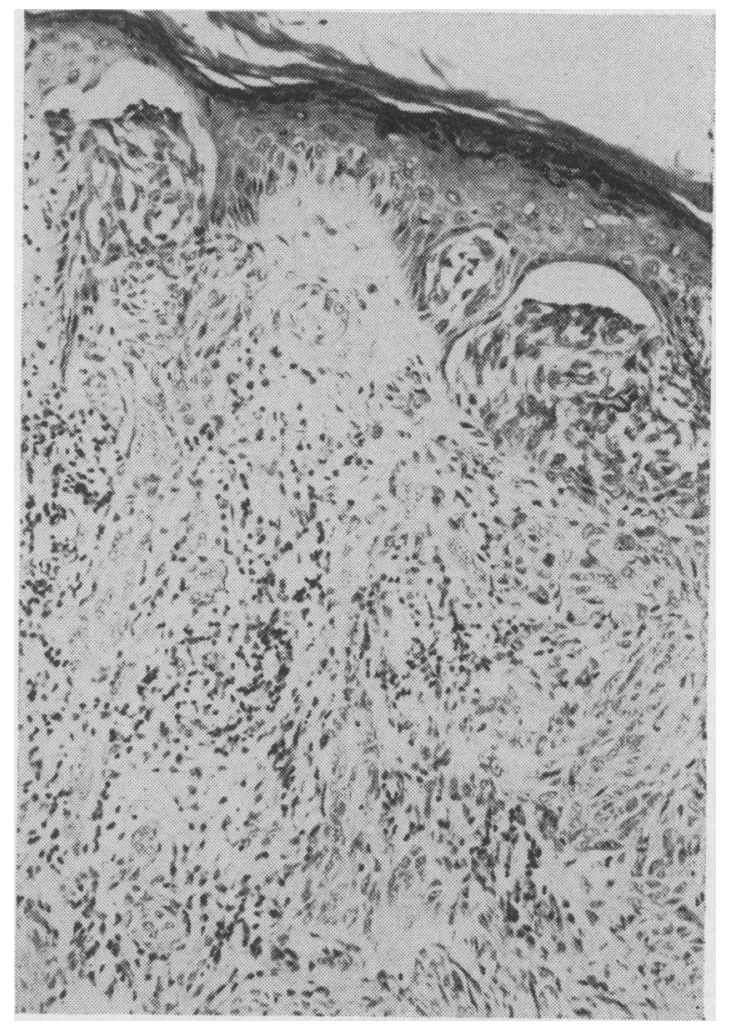

FIG. 9.- Juvenile melanoma of Spitz. A boy of I I years. Long history of skin tumour, no increase in size. Histology resembles malignant melanoma but is tidier (compare with Fig. 10). (3 18/49 $\times 172)$

the benign and the malignant forms becomes possible.

fuvenile Melanoma. The benign pre-pubertal melanoma of Spitz may resemble exactly a superficial malignant melanoma (Figs. 9 and 10). Sometimes, however, its invasion of the dermis is tidier, it does not appreciably destroy the dermal 


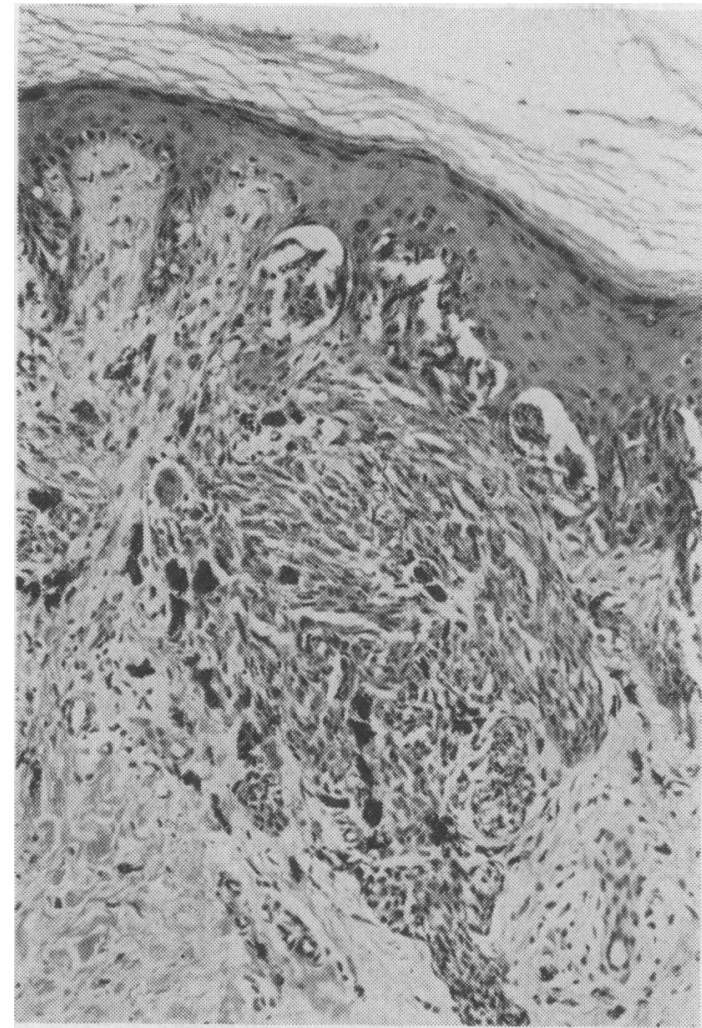

Fig. 10.-Edge of typical Stage 3 malignant melanoma with pigmented flare (compare with Fig. 9). $(261 / 49 \times 103)$

collagen, and the inflammatory reaction is less. The invading melanocytes sometimes get smaller centrifugally. Quite often multinucleate giant cells are present (as in benign melanomas, but not so often in malignant ones).

Blue Navi. The blue nævus does not usually give rise to any difficulty. It is a smooth, blueblack, dome-shaped lesion, usually about $0.5 \mathrm{~cm}$. diameter, composed of a well-defined but not circumscribed collection in the dermis of fusiform, usually heavily pigmented melanocytes mixed with fibrocytes and collagen fibres (Fig. I I). The difficulty is with the cellular variety of blue nævus (Allen and Spitz, I953). In this the spindly melanocytes are much bigger and appear active, though mitoses may be absent. There is, however, no disturbance of the pattern of dermal collagen (best seen on the cut surface with a hand lens in a good light) and no necrosis. Other useful considerations are the age of the patient (pre-pubertal nævi are nearly always benign), whether a blue nævus has been present from childhood, and whether it is occurring on one of the characteristic sites: buttocks, dorsum of hands or feet, face.

Non-Pigmented Malignant Melanoma. This is

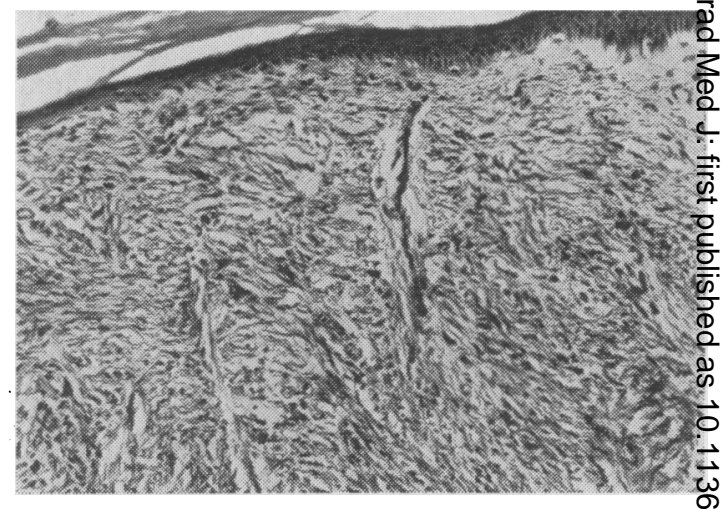

Fig. 1 I.-Typical blue nævus from a girl of to yearso $(96 / 50 \times 57)$

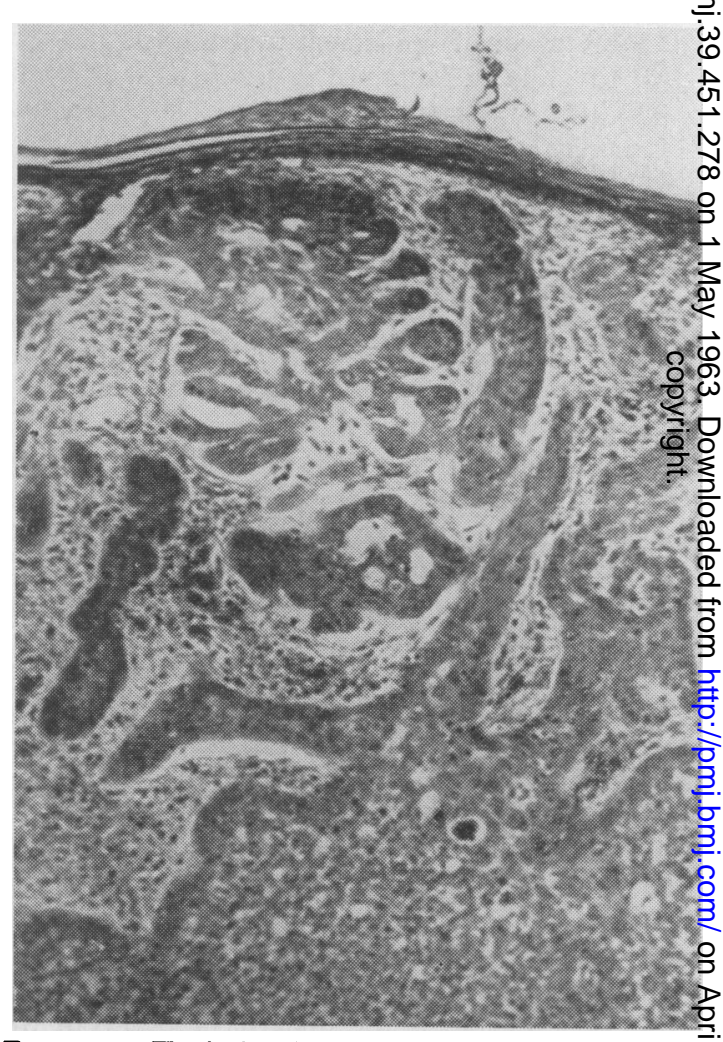

Fig. 12.-Typical pigmented basal-cell carcinom $(29 / 56 \times 137)$

sometimes misdiagnosed as an anaplastic squamouso carcinoma (Fig. 5). The mistake should be avoided as the prognosis is different. Often the origin of the tumour cells from the epiderma melanocytes may be seen. A search for melanion (stained by silver nitrate) may have reassuringo results.

Pigmented Basal-cell Carcinoma. The blaci rodent ulcer may look very like a malignan $\$$ 


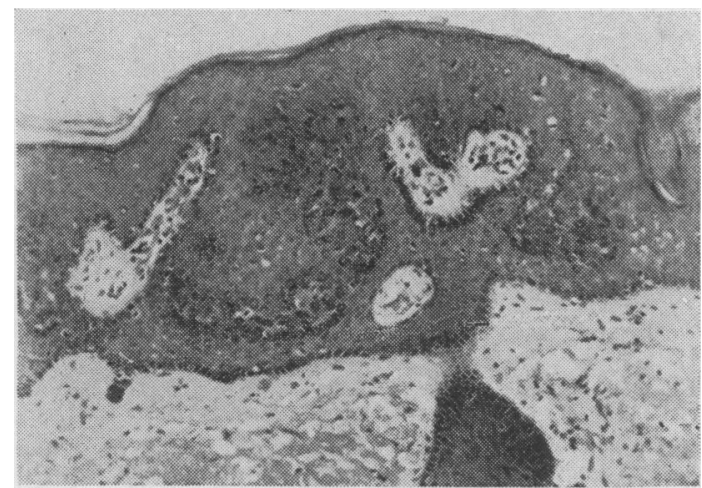

FIG. 13.-Verruca senilis (senile or seborrhœic wart). Pigmentation of basal layer. $(8 / 61 \times 82)$

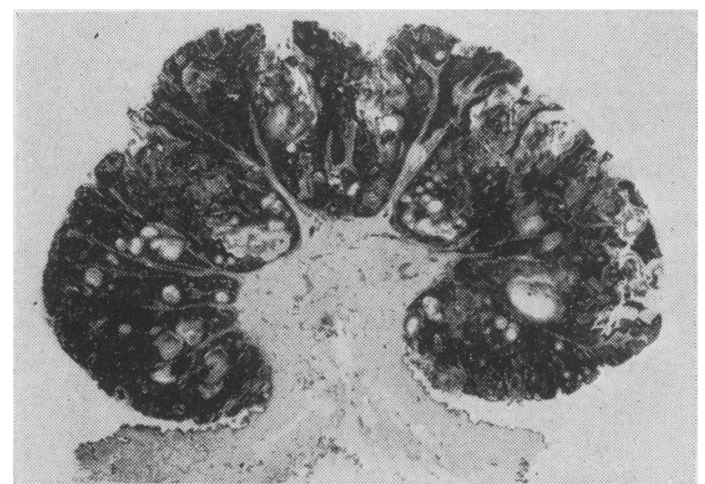

FIG. 14. Pigmented squamous papilloma. (P. M. $3784 \times 2.3)$

melanoma (Fig. 7), but it tends to have a nodular surface. There is no flare around it and the longer history often distinguishes it from a melanoma. Histologically, it should be easy. The club-shaped mass of cells with a palisade fringe make it one of the few types of tumour that are not mimicked by a melanoma (Fig. 12).

Senile Warts and Pigmented Papillomas. These two conditions are best considered together, as the latter commonly arises from the former. A senile wart when flat sometimes looks like a lentigo, but has a rough, keratinous surface and a biscuitlike brown colour. On section its true nature is apparent (Fig. 13). There is an overgrowth of the basal layers of the epidermis, with a general tendency for outward growth and hyperkeratosis. In the pigmented papilloma the hyperkeratosis is often intensified and may occur as cell nests (Fig. 14). The melanocytes, as in the black rodent and senile wart, are dendritic, hypertrophied and mainly basal in position.

Histiocytoma. This is a kind of dermal fibroma in which the cells behave like histiocytes, ingesting hæmosiderin and lipid. There is often melanosis of the overlying epidermis in the shape of a pig-

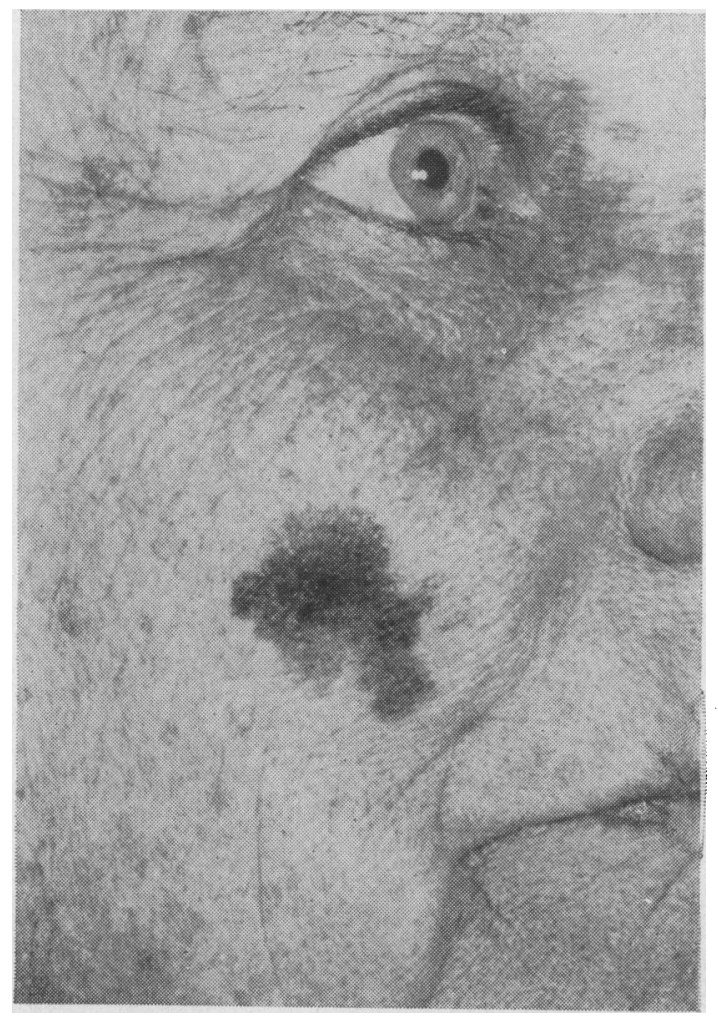

FIG. I 5.-Stage I malignant melanoma (lentigo maligna). (14/55, R. G.)

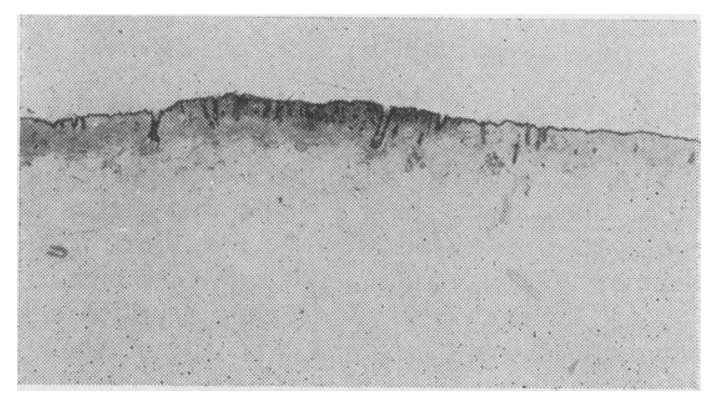

Fig. 16.-Section of Stage I malignant melanoma. Pigmented macrophages in inflamed dermis, but no invasion. (162/54 $\times 1.4)$

mented flare. The melanin is formed by the overactivity (but not hyperplasia) of the epidermal melanocytes.

\section{Histological Prognostic Aids}

A. Stage of Development of Malignant Melanoma Stage I: No invasion of dermis.

Stage 2: Invasion of superficial dermis but no tumour formation.

Stage 3: Tumour formation.

A Stage I malignant melanoma is a flat brown patch undergoing progressive enlargement, often 


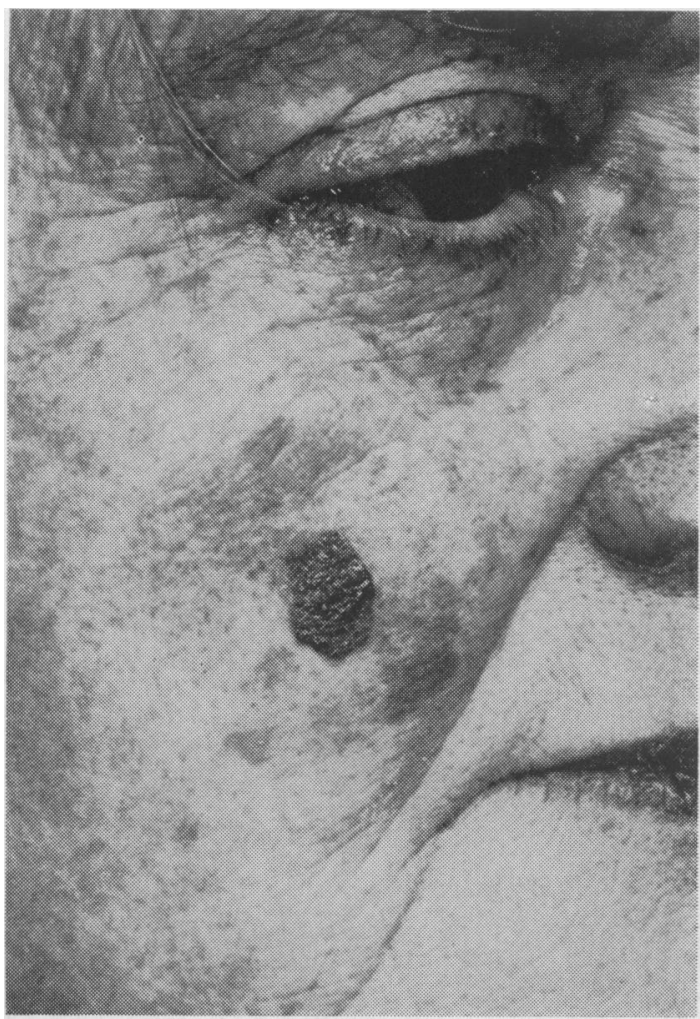

Fig. 17.-Stage 2 malignant melanoma. The whole area is malignant but only the central area invasive. $(86 / 57$, C. J.)

with a marked colour change, but in which the melanocytes have not yet invaded the dermis (Figs. 3, 15 and 16). The practical point of this is that they cannot have invaded lymphatics or venules and so metastases do not form. The lesion is a kind of 'carcinoma in situ'. The melanocytes are enormous, usually hyperpigmented and form epidermal buds. There is usually inflammation of the dermis.

In a Stage 2 malignant melanoma there is invasion of the dermis but no tumour formation (Figs. 17 and 18). Lymphatic invasion is therefore possible but is uncommon, so that in uncomplicated cases lymph node dissection is unnecessary, provided adequate primary excision is achieved.

In a Stage 3 malignant melanoma (Figs. I9 and 20 ) there is tumour formation and metastases are common.

The occurrence of metastases in the series of 220 cases published by Petersen and others ( 1962 ) and the five-year survival rate in their 93 determinate cases is shown in the following table in relation to the stage of the melanoma.

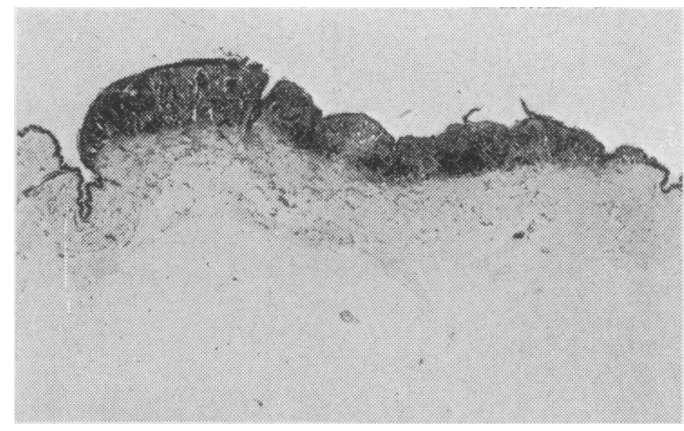

FIG. I8.-Section of Stage 2 malignant melanoma Heavily pigmented melanocytes invade the dermis but do not form a tumour. (42/56 x 4)

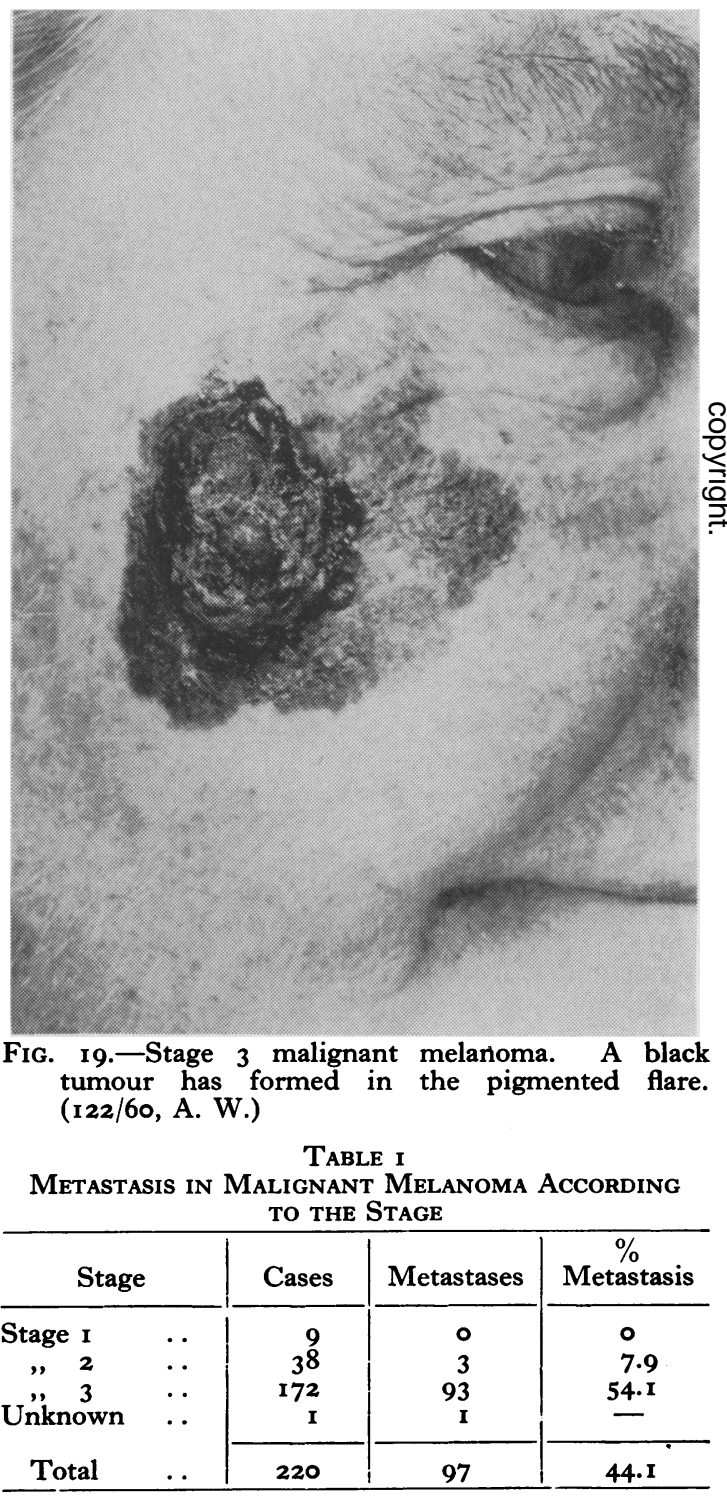




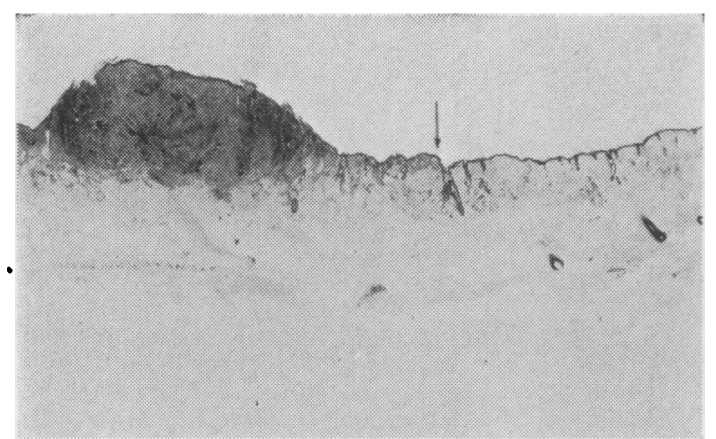

FIG. 20.-Section of Stage 3 malignant melanoma. Pigmented flare with tumour formation. Arrow points to margin of flare. $(54 / 57 \times 3)$

TABLE 2

Five-year Survival Rates of Melanoma According TO THE Stage

\begin{tabular}{|c|c|c|c|c|}
\hline Stage & & $\begin{array}{c}\text { Determinate } \\
\text { Cases }\end{array}$ & $\begin{array}{l}\text { Alive After } \\
\text { Five Years }\end{array}$ & $\%$ \\
\hline $\begin{array}{cc}\text { Stage } 1 \\
\Rightarrow \quad 2 \\
\text { Unknown }\end{array}$ & $\begin{array}{l}\cdots \\
\cdots \\
\cdots\end{array}$ & $\begin{array}{r}4 \\
17 \\
71 \\
1\end{array}$ & $\begin{array}{r}4 \\
14 \\
36 \\
0\end{array}$ & $\begin{array}{c}100 \\
82.3 \\
50.7 \\
0\end{array}$ \\
\hline Total & .. & 93 & 54 & 58.1 \\
\hline
\end{tabular}

These tables show the value of using this method of staging both as a prognostic aid and as a guide to treatment, since in Stages $I$ and 2 it will not usually be necessary to carry out removal of the regional lymph nodes.

\section{B. Obvious Invasion of Dermal Lymphatics Near the Primary}

It is always worth examining carefully the surroundings of the primary tumour to see if there is any obvious invasion of the dermal lymphatics, as this makes the prognosis much worse, indicating the likelihood that the local lymph nodes have already been invaded and the possibility that lymphatic metastases may arise in the dermis at some point between the lymph nodes and the primary. The observation of dermal lymphatic invasion is never made with superficial melanomas in Stage $I$ and rarely in those of Stage 2, so the following table relates only to Stage 3 determinate cases.

TABLE 3

Prognosis of Stage 3 Determinate Cases

(In relation to the presence of obvious invasion of the dermal lymphatics in the primary tumour.)

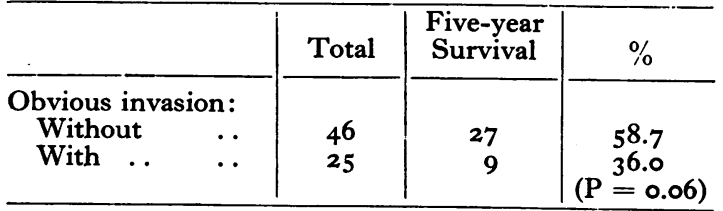

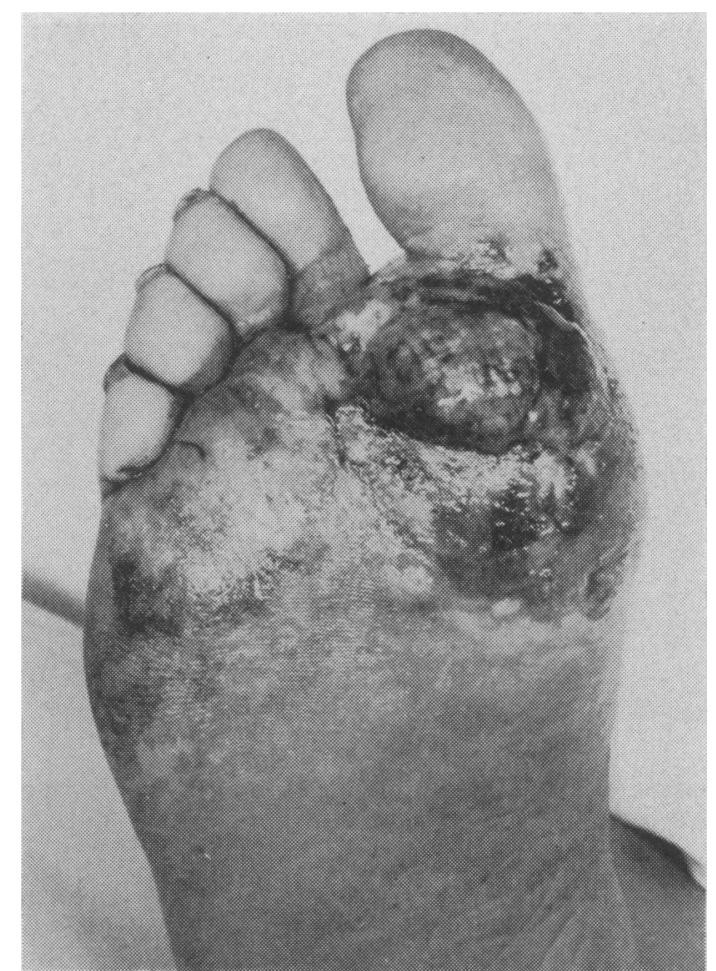

Fig. 21.-Stage 3 malignant melanoma of foot with ulcerating tumour and extensive pigmented flare. The whole area is malignant. (1956, A. P.)

This table shows that the five-year survival rate is much worse in those cases where invasion of the dermal lymphatics has been observed near the primary tumour.

\section{Effects of Trauma (Including Incisional Biopsy) on Melanomas}

Analysis by the authors of their 220 cases of malignant melanoma revealed without doubt that those lesions which had suffered trauma in any way had a much worse prognosis than those not so damaged. Much of the trauma was the result of wrong treatment, namely, curettage, cauterization, diathermy coagulation, carbon dioxide snow, freezing and incisional biopsy, generally based on incorrect diagnosis and ignorance of the risks involved. Trauma excites an inflammatory reaction, increasing blood supply and tissue tension, so stepping up the outflow of migratory cells via the lymphatic and venous channels.

\section{Behaviour of Melanomas}

Treatment is based on an understanding of the behaviour of these tumours. The pigmented flare commonly seen around a melanoma is malignant (Fig. 21) and this malignant potential extends beyond the visible zone of pigment. This area is 


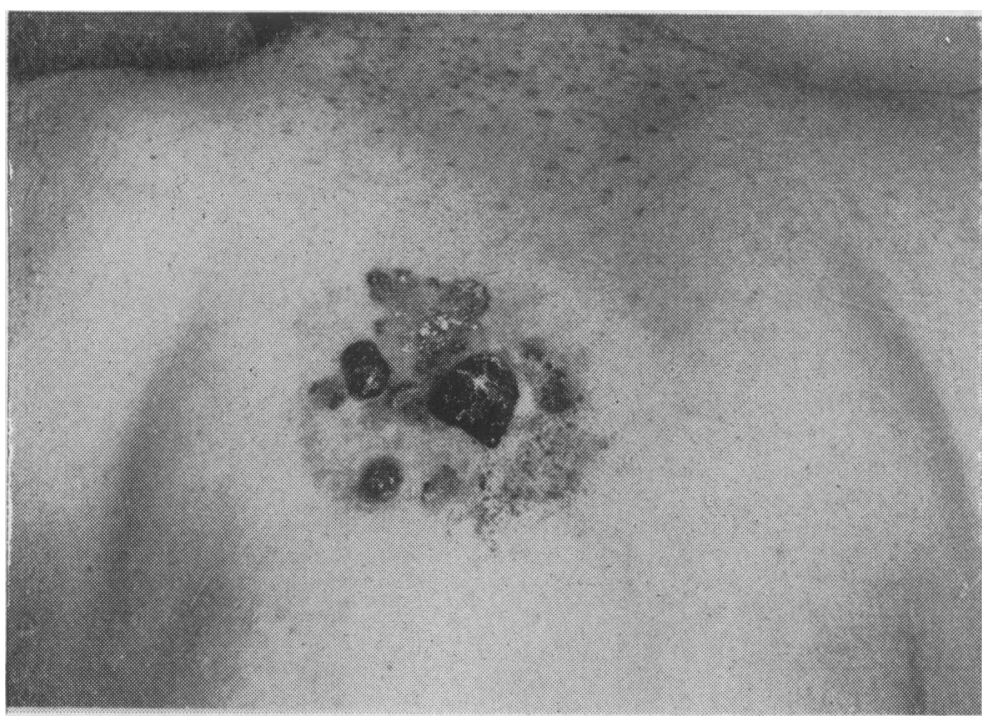

FIG. 22.-Malignant melanoma of back with epidermal and lymphatic satellites. Metastasis to left axilla. (39/56, S. M.).

expanding as the lesion progresses. Spread beyond this zone may take the form of satellites, which are seedling growths found around the primary lesion with an intervening zone of apparently normal skin. They may arise in one of two ways: either they arise from the epidermal melanocytes and are an example of the discontinuous spread of malignant melanocytes through the epidermis or they may arise by direct spread through the dermal lymphatics and so may appear at any distance from the primary, but usually within a radius of 5 to $10 \mathrm{~cm}$. (Fig. 22). In either case the satellites may not have reached macroscopic proportions by the time of initial treatment and this undoubtedly accounts for the very high incidence of recurrences which occur in and around the scar following inadequate excision.

One of us (O. C. L.) always searches for and reports on the state of the dermal lymphatics. If these are obviously invaded by melanoma cells, then distant spread along the lymphatic channels is much more likely to take place. At the time of any surgical procedure designed to eliminate the malignant melanoma some lymphatics may well contain metastatic cells en route between the primary and the regional lymph nodes. Where the primary and the regional lymph nodes are in reasonable proximity to each other it is rational to carry out a monobloc excision in continuity. In many instances, however, the distance between the primary and the regional lymph nodes is such that this would be too great an operation and the general opinion is that it is not then worth while. There is, however, some evidence that if there is an interval between the excision of the primary and the regional lymph nodes then the 'pipeline' may empty and the migratory cells reach the lymph nodes where they can be dealt with by radical clearance.

A study of many recurrent tumours following surgical excision has formed the basis of the recommendations for primary surgery.

The prognosis of cases so treated is much more favourable than generally supposed and fully justifies these measures which will be described.

There will always be cases which behave in a totally unpredictable manner. On the one hand there are those with obvious clinical and microscopical spread which remain free of disease for many years and on the other those which look favourable and which behave badly. The experienced clinician will have had occasional experience of some cases of cancer which undergo spontaneous regression and the malignant melanoma is no exception to this. Undoubtedly these tumours can respond to certain changes in naturally occurring hormones, and Bullen, Freundlich, Hale, Marshall and Tudway (1963) have been able to demonstrate this fact by estimation of the $32 \mathrm{P}$ uptake under varied conditions of hormonal balance. They write more fully elsewhere in this journal discussing the useful application of this test.

It is an often repeated observation that a melanoma which recurs a number of times may do so at irregular intervals and hope should never be abandoned. We advocate that every recurrence should be treated with the same care and thoroughness as a fresh primary. One patient had a primary melanoma of the leg which recurred three times at intervals of one year. The fourth time seven separate nodules were counted on the skin of the 


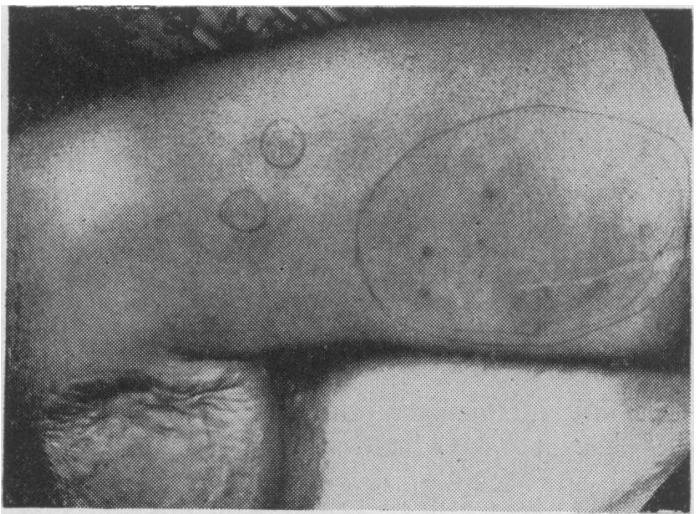

FIG. 23.-Multiple skin recurrences in thigh following wide excision of primary melanoma of calf and block dissection of inguinal lymph nodes. (1957, R. L.)

thigh (Fig. 23); these were excised widely. The defect, approximately $25 \times 15 \mathrm{~cm}$., was skingrafted and now three years later there is still no sign of trouble (Fig. 24). Another similar case, a woman of 42 , produced a succession of regional deposits, finally developing several local and one distant skin metastases, all of which were excised; she has been free of trouble for over two years.

In the course of routine follow-up examinations some doubtful nodules will be picked out. It is our practice to excise these routinely. One such case produced three successive small dusky nodules in the leg close to the margin of the skin graft. These turned out to be merely small thrombosed veins. Then a fourth lesion appeared and was excised. This turned out to be a true recurrence.

\section{Melanoma and Pregnancy}

This is a relationship which cannot be clearly established because the number of females with melanoma who become pregnant is too small for statistical study. However, the general impression is that the disease tends to be activated by pregnancy although the overall prognosis is not necessarily worse.

\section{Surgical Treatment}

It must be emphasized that from the first moment a malignant melanoma is suspected it must be protected from injury until elective treatment is carried out and this must be performed with the minimum of delay. Meddlesome surgery is inexcusable and all procedures should be in the hands of a competent surgeon who is not afraid to carry out the necessary wide excisions and immediate skin grafting on the raw surfaces.

\section{Ansesthesia}

It is our practice to carry out all surgery of

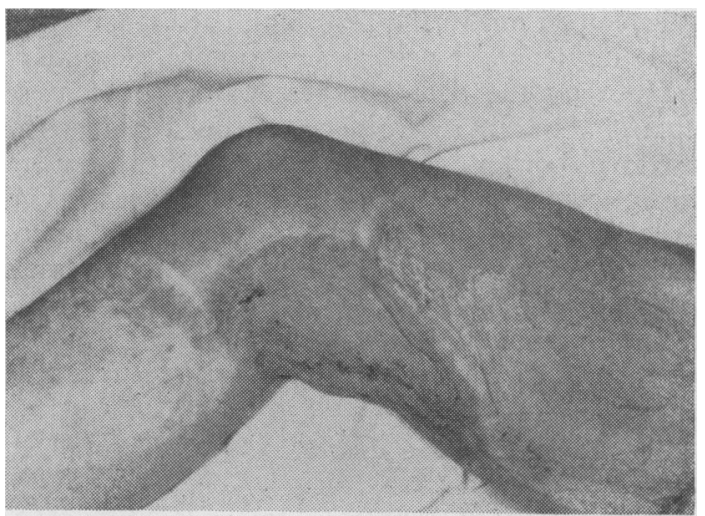

FIG. 24.-The same case as Fig. 23 three years later, following excision of recurrences and skin graft. There have been no recurrences since then. (1960, R. L.)

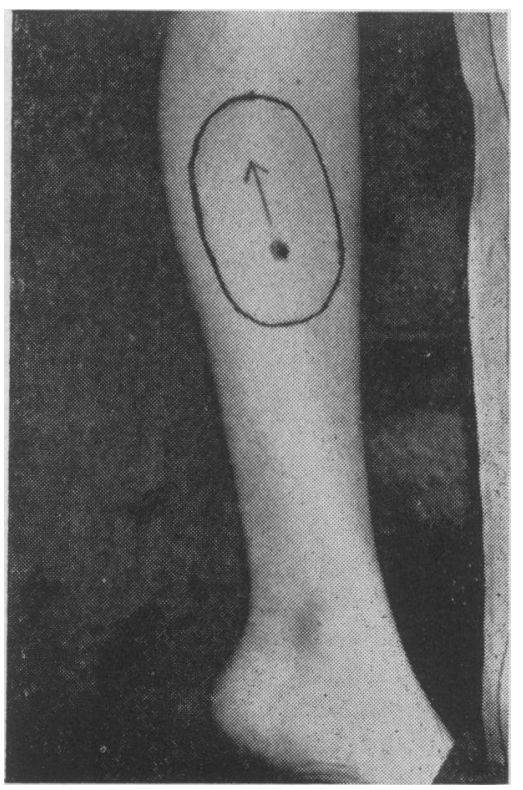

FIG. 25.- Stage 2 malignant melanoma of leg. The area of eccentric excision has been mapped out, and the arrow indicates the direction of lymph flow. (34/63, M. F. B.)

melanomas under general anæsthesia unless the patient is unfit, in which case local anæsthesia is used. It is believed that the rise in intercellular pressure from injection of local anæsthetic agents can promote cell embolism by forcing cells beyond the operating field (Young, Lumsden and Stalker, 1950).

\section{Surgery of the Primary Lesion}

The lesion must be treated with the greatest care and gentleness and on no account should an Esmarch compression tourniquet be used. The area selected for excision is carefully estimated 
and mapped out in ink (Fig. 25), the extent being solely influenced by the desire to cure the patient and not by the desire to lessen the problem of repair. The incision is commenced along the proximal border, carried down to the intended depth, usually beneath the deep fascia, and planned to finish distally. Meticulous hæmostasis is achieved by ligature of the larger vessels and diathermy coagulation of the rest.

The following optimum extents of excision have been determined by studying results of a large series of cases.

\section{Malignant Melanoma}

Stage I. I cm. margin.

Stage 2. Eccentric excision (Fig. 25), including underlying deep fascia of $5 \mathrm{~cm}$. on the narrowest rnargin distally, and $6 \mathrm{~cm}$. on each side and $7 \mathrm{cn}$. on the proximal side; the wider margin on this side takes into account the direction of natural lymphatic flow. If histological examination of the melanoma shows lymphatic permeation then a prophylactic lymph node dissection is carried out two or three weeks later.

Stage 3 A similar eccentric excision is carried out, but is wider, the margins being 6,8 and 15 $\mathrm{cm}$. and followed by delayed prophylactic block dissection of the regional lymph nodes. The operation is carried out simultaneously and in continuity when the primary and the lymph nodes are close together.

Split skin grafts are used to cover these extensive raw surfaces and since such a graft is available in any quantity the size of the defect does not matter. They should never be taken from the same limb but always from an area remote from the primary. Transposition and rotation flaps are used only occasionally to repair defects on the face, which is dealt with as a separate site. Grafting of particular areas, such as between the scapulæ, on the chest wall, perineum, buttocks, etc., present technical difficulties which can be readily overcome by adopting a different grafting technique. The graft is taken, wrapped in a moist saline swab, and stored at $\mathrm{I}^{\circ} \mathrm{C}$. in a sealed sterile container. The raw surface is dressed temporarily with a non-adhesive dressing and on the third or fourth day, with the full co-operation of the patient and as a ward procedure, the graft is applied, with the patient resting comfortably in bed, in the manner of a simple dressing. The graft is neither stitched nor covered, being protected by a bed cradle. The grafts vascularize quickly and after seven days may be safely dressed and the patient allowed full mobility.

The Foot. Owing to the poor prognosis widest possible excision must be carried out and it is fortunate that split skin grafts on the sole of the foot are quite adequate and stand up well to ordinary wear and tear and may be used with confidence. However, such grafts do require three to four weeks before weight bearing is allowed and need special protection against injury until they have settled in, lost their increased vascularity and gained some sensation. In all grafting procedures surplus skin should be stored in the same manner in a refrigerator so that it may be used to re-graft any areas which might have failed.

The Face. The face does not lend itself to the same extent of radical surgery as the leg or trunk. Fortunately, however, it is one of the most favourable sites to be affected and less radical surgery can be carried out. In Stage I and 2 lesions and early Stage 3 lesions in older persons a margin of $2 \mathrm{~cm}$. should be aimed at. With the more advanced Stage 3 lesions, particularly in younger persons with the more active type of primary, the most radical excision that is possible should be carried out, together with dissection of the pre-auricular and cervical lymph nodes in continuity if possible. The superficial lobe of the parotid gland containing lymph nodes is removed, preserving the full function of the 7 th nerve. Certain minute lesions which conform to Stages $I$ and 2 and occur on the eyelids have done well following excisions with a margin of $\mathrm{I} \mathrm{cm}$.

Fingers and Toes. When a finger or toe is the site of a primary it should be amputated and skin and deep fascia removed proximally according to the previous recommendations.

Trunk. The prognosis at this site is poor and the maximum possible area of excision should be carried out in all cases. Spread is in the direction of the nearest lymph nodes and midline lesions spread to both sides. Surgery should be planned accordingly.

Regional Lymph Nodes. Block dissection, as indicated above, should be carried out two to three weeks after excision of the primary, in order to allow the lymphatics in between to clear themselves of migrating malignant cells. If the lymph nodes are palpably enlarged, then the dissection should be extended to include the next group of nodes. Simultaneous dissection should be carried out only when in continuity with the primary. In all cases block dissection should be carried out as a radical procedure in accordance with the standard practice.

Surgery of Recurrences. All recurrences should be treated with urgency and the same thoroughness as a primary. Hope should never be abandoned even when a cluster of nodules appears on several occasions. 


\section{Chemotherapy}

Drugs of the nitrogen mustard type, particularly melphalan and the antimetabolite methotrexate, have been used in the treatment of recurrent melanoma of the limbs. Melphalan has been given by continuous perfusion using a pump oxygenator (Creech, Krementz, Ryan and Winblad, 1958) with transient success. Methotrexate has been given by slow intra-arterial infusion (Espiner, Vowles and Walker, 1962). Experience has shown that the risks and disturbance to the patient are not matched by the benefit and such treatment has no place in the routine management of malignant melanoma of the limbs. Work is still going on and the results of treating face and head lesions are a little more encouraging.

\section{Hormonal Therapy}

There is evidence that some women in the forties who have a definite pattern of recurrence undergo a favourable change in tumour growth behaviour as they approach the menopause. A small percentage have benefited by oophorectomy and others by hypophysectomy.

In a very few instances males have responded temporarily to therapy with œstrogen antagonists.

\section{Summary}

Much of the improvement in the prognosis of malignant melanoma of the skin is attributable to early detection with precise diagnosis, adequate primary treatment and the elimination of meddlesome forms of interference. Errors in diagnosis are usually mistaking a malignant melanoma for something else, or, less commonly, mistaking another lesion for a malignant melanoma. The ways in which these errors may be eliminated have been discussed.

Certain guides to prognosis and treatment are described. A Stage I melanoma (malignant, but not invading dermis) does not metastasize. A Stage 2 melanoma (invasion of dermis, but no tumour formation) rarely does so, while a Stage 3 melanoma (tumour formation) metastasizes in more than half the cases. Obvious invasion of dermal lymphatics near the primary is of bad prognostic significance. Many cases later developing lymphatic skin metastases have shown this phenomenon. Deliberate trauma of any sort to a melanoma is to be condemned. The zone of malignant change may extend outside the visible pigmented flare and satellites may occur.

The principles of surgical treatment are described. They include excision of a much wider zone of apparently normal skin than is commonly practised, and this should be widest in the direction of lymphatic flow. By using margins of the widths recommended for the different sites, most recurrences and lymphatic skin metastases may be avoided. Delayed prophylactic lymph node dissection is recommended for Stage 3 malignant melanomas and for any that show obvious invasion of the dermal lymphatics, even if the nodes are not clinically enlarged. Split skin grafts can be taken at the time of the operation (not from the same limb), stored at $I^{\circ} \mathrm{C}$. and applied as a ward dressing on the third or fourth post-operative day. Every recurrence should be treated as thoroughly as if it were a new primary. Hope need never be abandoned.

We are grateful to Mr. L. G. Banham and-Mr. D. N. White for the photography and to the Editor of the British Fournal of Plastic Surgery for permission to use material and some illustrations from our previously published work.

The histological details presented in this paper are being amplified for the chapter on 'The diagnosis of melanoma of the skin', in the next edition of 'Recent Advances in Clinical Pathology', now in the Press.

\section{REFERENCES}

Allen, A. C., and SpItz, S. (1953): Malignant Melanoma: A Clinico-Pathological Analysis of the Criteria for Diagnosis and Prognosis, Cancer, 6, I.

Block, G. E., and Hartwell, S. W., Jr. (r96r): Malignant Melanoma: A Study of 217 Cases, Ann. Surg. (Suppl.), I54, 74 .

Bullen, M. A., Freundlich, H. H., Hale, B. T., Marshall, D. H., and Tudway, R. T. (1963): The Activity of Malignant Tumours and Response to Therapeutic Agents Studied by Continuous Records of Radioactive Phosphorus Uptake, Postgrad. med. F., 39, 265.

Clemmesen, J., and Schultz, G. (1960): Cancer Incidence in Denmark, i 953 to r957, Dan. med. Bull., 7, r68.

Creech, O., Jr., Krementz, E. T., Ryan, R. F., and Winblad, J. N. (1958): Chemotherapy of Cancer: Regional Perfusion Utilizing an Extracorporeal Circuit, Ann. Surg., 148, 616.

Espiner, H. J., Vowles, K. D. J., and WALker, R. M. (1962): Cancer Chemotherapy by Intra-arterial Infusion, Lancet, $\mathrm{i}, \mathrm{I} 77$.

Ochsner, A., and Harpole, D. H. (1962): Malignant Melanoma: Its Prognosis as Influenced by Therapy, Ann. Surg., I55, 629 .

Olsen, G. (I96r): Maligne Melanomer i Huden, Ugeskr. Lag., 123, 1235.

Pack, G. T., Scharnagel, I. M., and Gerber, D. M. (1953): The Treatment of Malignant Melanoma of the Skin, Surg. Clin. N. Amer., p. 5 I 7.

- (1959): End Results in the Treatment of Malignant Melanoma, Surgery, 46, 447.

Petersen, N. C., Bodenham, D. C., and Lloyd, O. C. (1962): Malignant Melanomas of the Skin, Brit. F. plast. Surg., I5, $49,97$.

Spitz, S. (1948): Melanomas of Childhood, Amer. Y. Path., 24, 591.

White, L. P. (1959): Studies on Melanoma, II : Sex and Survival in Human Melanoma, New Engl. F. Med., 260, 289.

Young, J. S., Lumsden, C. E., and Stalker, A. L. (1950): The Significance of the 'Tissue Pressure' of Normal Testicular and of Neoplastic (Brown-Pearce Carcinoma) Tissue in the Rabbit, F. Path. Bact., 62, 3 I3. 